\title{
Seroprevalence and Risk Factors of Chlamydia Infection in Domestic Rabbits (Oryctolagus cuniculus) in China
}

\author{
Xiaoting Ni, ${ }^{1,2}$ Siyuan Qin, ${ }^{2,3}$ Zhilong Lou, ${ }^{2,3}$ Hongrui Ning, ${ }^{2,4}$ and Xiaolin Sun ${ }^{1}$ \\ ${ }^{1}$ College of Veterinary Medicine, Gansu Agricultural University, Lanzhou, Gansu 730070, China \\ ${ }^{2}$ State Key Laboratory of Veterinary Etiological Biology, Lanzhou Veterinary Research Institute, \\ Chinese Academy of Agricultural Sciences, Lanzhou, Gansu 730046, China \\ ${ }^{3}$ College of Animal Science and Technology, Jilin Agriculture University, Changchun, Jilin 130118, China \\ ${ }^{4}$ College of Animal Science and Technology, Anhui Agricultural University, Hefei, Anhui 230000, China
}

Correspondence should be addressed to Xiaolin Sun; sunxl@gsau.edu.cn

Received 6 February 2015; Revised 14 March 2015; Accepted 23 March 2015

Academic Editor: Kent M. Reed

Copyright (C) 2015 Xiaoting Ni et al. This is an open access article distributed under the Creative Commons Attribution License, which permits unrestricted use, distribution, and reproduction in any medium, provided the original work is properly cited.

Chlamydia spp. are obligate intracellular bacteria distributed all over the world, known to cause various forms of diseases in animals and humans. In the present study, a serological survey was conducted to detect the seroprevalence and risk factors associated with rabbit chlamydiosis in northeast China, including Liaoning province, Jilin province, Heilongjiang province, and Inner Mongolia Autonomous Region. Antibodies to Chlamydia were determined by indirect hemagglutination assay (IHA). The overall seroprevalence was estimated at $17.88 \%$ in total of 800 blood samples. The Chlamydia seroprevalence varied in domestic rabbits from different factors, and genders of domestic rabbits were considered as major risk factors associated with Chlamydia infection. Our study revealed a widespread and high prevalence of Chlamydia infection in domestic rabbits in northeast China, with higher exposure risk in female domestic rabbits. These findings suggested the potential importance of domestic rabbits in the transmission of zoonotic Chlamydia infection, and thus Chlamydia should be taken into consideration in diagnosing rabbit diseases. To our knowledge, there is no report of Chlamydia infection in domestic rabbits in China and the results extend the host range for Chlamydia, which has important implications for public health and the local economy.

\section{Introduction}

Chlamydia is a genus comprising important zoonotic obligate intracellular pathogens that affect humans and a wide range of animals $[1,2]$. Chlamydia infection can cause various diseases in nonhuman mammals and birds, including conjunctivitis, atypical pneumonia, enteritidis, endocarditis, and even abortion, resulting in serious economic losses [3-6]. Several Chlamydia species are known to infect humans and are of serious public health significance because they may result in atherosclerosis, coronary heart disease, pneumonia, and other severe diseases [7]. Chlamydia abortus and Chlamydia psittaci are of particular importance because they can cause abortion and psittacosis, respectively, in animals, birds and humans. In Egypt, a study revealed that in symptomatic gynecologically diseased women the seroprevalence of Chlamydia psittaci was $50.0 \%$, Chlamydia abortus was $35.7 \%$, and
Chlamydia trachomatis was $15.2 \%$ [8], and in the genital tract of cattle the main Chlamydiaceae were Chlamydia psittaci and Chlamydia abortus [9]. The animal diseases caused by these microorganisms should be given more attention due to their zoonotic potential $[1,7,10]$.

The breeding of domestic rabbits (Oryctolagus cuniculus) for human consumption has a long radition in China, and rabbit industry is one of green economy industries, which is emerging and promising, with broad space for development and has gone through a long glorious history and its products have brought great wealth to the rural people in China. At present, China plays an important role of breeding rabbits, and domestic rabbits yield high quality meat and hair which are transported to many parts of home and abroad. However, disease is the main challenge for rabbit industry [11, 12]. To date, there is little information about the seroprevalence of Chlamydia and the risk factors associated with Chlamydia 
TABLE 1: Seroprevalence of Chlamydia infection in domestic rabbits by age, gender, and breed in China, as detected by indirect haemagglutination assay (IHA).

\begin{tabular}{lcccc}
\hline Factor & Category & Number examined & Number positive & \% (95\% CI) \\
\hline \multirow{2}{*}{ Age } & Juvenile & 464 & 92 & $19.83(16.20-23.46)$ \\
& Adult & 336 & 51 & $15.18(11.34-19.02)$ \\
\hline \multirow{2}{*}{ Gender } & Male & 319 & 45 & 98 \\
& Female & 481 & 52 & $14.11(10.29-17.93)$ \\
\multirow{3}{*}{ Breed } & Chinese White Rabbit & 249 & 38 & $20.84(16.78-23.97)$ \\
& California Rabbit & 250 & 53 & $15.20(10.75-19.65)$ \\
& New Zealand Rabbit & 301 & 39 & $17.61(13.31-21.91)$ \\
\hline \multirow{4}{*}{ Region } & Liaoning province & 200 & 41 & $19.50(14.01-24.99)$ \\
& Jilin province & 200 & 33 & $20.50(14.91-26.10)$ \\
& Heilongjiang province & 200 & 30 & $16.50(11.36-21.64)$ \\
Total & Inner Mongolia Autonomous Region & 200 & 143 & $15.00(10.05-19.95)$ \\
\hline
\end{tabular}

infection in domestic rabbits in the world. Thus, the present study was aimed at determining the seroprevalence of Chlamydia infection in domestic rabbits in northeast China and identifying factors associated with the presence of Chlamydia antibodies in domestic rabbits in the study area.

\section{Materials and Methods}

2.1. Ethics Statement. This study was approved by the Animal Ethics Committee of Lanzhou Veterinary Research Institute, Chinese Academy of Agricultural Sciences (Approval no. LVRIAEC2013-010). The domestic rabbits, from which the serum samples were collected, were handled in accordance with good animal practices required by the Animal Ethics Procedures and Guidelines of the People's Republic of China.

2.2. Collection and Preparation of Serum Samples. A total of eight hundred domestic rabbits were randomly sampled from Liaoning province, Jilin province, Heilongjiang province, and Inner Mongolia Autonomous Region. From each rabbit, approximately $2 \mathrm{~mL}$ of blood was collected from the marginal ear vein or postmortem. After centrifugation at 3,000 rpm for $10 \mathrm{~min}$, sera were collected and stored at $-20^{\circ} \mathrm{C}$ until use. Handling of rabbits was in compliance with the Animal Ethics Procedures and Guidelines of the People's Republic of China. Information about species, ages, geographic origin, and gender was acquired from breeders.

2.3. Serological Examination. Antibodies to Chlamydia were tested by indirect hemagglutination antibody (IHA) using a commercially marketed kit (Lanzhou Veterinary Research Institute, Chinese Academy of Agricultural Sciences, Lanzhou, Gansu Province, China). The procedures are according to the manufacturer's instructions and previous descriptions $[13,14]$. The IHA kit was performed following the standard protocol recommended by Chinese Center for Animal Diseases Prevention and Control, and the sensitivity and specificity of the test are $100 \%$ and $95 \%$, respectively [13]. The sensitivity and specificity values for the testing kit used in this study have been validated by The Ministry of Agriculture of the People's Republic of China (NY/T 562-2002). In brief, $75 \mu \mathrm{L}$ of the IHA dilution solution was transferred into 96 well V-bottomed polystyrene plates with $25 \mu \mathrm{L}$ of serum added and diluted in a fourfold series from $1: 4$ to $1: 64$. The plates were shaken for $2 \mathrm{~min}$ and then incubated at $37^{\circ} \mathrm{C}$ for $2 \mathrm{~h}$ without shaking. The test was considered positive when a layer of agglutinated erythrocytes was formed in wells at dilutions of $1: 16$ or higher, and positive and negative controls were included in each test. Those sera which showed dubious results were picked out for retest.

2.4. Statistical Analyses. Regions, genders, age groups, and breeds of rabbits were analyzed in a multivariable logistic regression model, and probability $(P)$ value $<0.05$ was considered as statistically significant. Odds ratios (OR) with 95\% confidence intervals are also calculated. All statistical analyses were performed using the PASW Statistics 18.0 (SPSS Inc., IBM Corporation, Somers, NY).

\section{Results}

Antibodies to Chlamydia were detected in 143 of 800 (17.88\%, 95\% CI 15.22-20.53) domestic rabbits. Of these, antibody titers were $1: 16$ in $22,1: 32$ in $18,1: 64$ in $33,1: 128$ in $30,1: 256$ in 27, 1:512 in 9, and 1:1024 in 4, respectively (Table 3 ). The Chlamydia seroprevalence varied in domestic rabbits from different regions, ranging from $15.00 \%$ (95\% CI 10.05-19.95) to $20.50 \%$ (95\% CI 14.91-26.10) (Table 1), but the differences were not significantly different. The juvenile rabbits had higher seroprevalence (19.83\%) than that in adults (15.18\%). The seroprevalence of Chlamydia was diverse in different breeds of domestic rabbits; the highest level was $20.88 \%$ in Chinese White Rabbits, followed by $17.61 \%$ in New Zealand Rabbits and $15.20 \%$ in California Rabbits (Table 1). However, the differences were not statistically significant $(P>0.05)$.

In terms of gender, seroprevalence of Chlamydia in female $(20.34 \%)$ was higher than that in male $(14.11 \%)$, and gender was considered as major risk factors associated with Chlamydia infection in domestic rabbits $(P<0.05$, Table 2$)$. And the risk of Chlamydia infection in female was more than 
TABLE 2: Odds ratios for genders of domestic rabbits are taken as risk factors for Chlamydia seroprevalence in domestic rabbits.

\begin{tabular}{lccccc}
\hline Gender & Number tested & Number positive & Prevalence (\%) & OR (95\% CI) & $P$ value \\
\hline Male & 319 & 45 & $14.11(10.29-17.93)$ & Reference \\
Female & 481 & 98 & $20.34(16.78-23.97)$ & $1.56(1.06-2.29)$ & 0.024 \\
\hline
\end{tabular}

TABLE 3: Seroprevalence and antibody titers of Chlamydia infection in domestic rabbits, as detected by indirect haemagglutination (IHA) test.

\begin{tabular}{|c|c|c|c|c|c|c|c|c|c|c|c|}
\hline \multirow{2}{*}{ Biometric data } & \multirow{2}{*}{ Category } & \multicolumn{7}{|c|}{ Antibody titers } & \multirow{2}{*}{$\begin{array}{l}\text { Number } \\
\text { positive }\end{array}$} & \multirow{2}{*}{$\begin{array}{c}\text { Number } \\
\text { tested }\end{array}$} & \multirow{2}{*}{$\begin{array}{c}\text { Prevalence } \\
(\%)\end{array}$} \\
\hline & & $1: 16$ & $1: 32$ & $1: 64$ & $1: 128$ & $1: 256$ & $1: 512$ & $1: 1024$ & & & \\
\hline \multirow{2}{*}{ Gender } & Female & 17 & 16 & 20 & 18 & 16 & 7 & 4 & 98 & 319 & 20.34 \\
\hline & Male & 5 & 2 & 13 & 12 & 11 & 2 & 0 & 45 & 481 & 14.11 \\
\hline \multirow{3}{*}{ Age } & Infancy & 6 & 6 & 8 & 13 & 6 & 2 & 1 & 42 & 175 & 24.00 \\
\hline & Juvenile & 8 & 6 & 12 & 9 & 10 & 4 & 1 & 50 & 289 & 17.30 \\
\hline & Adult & 8 & 6 & 13 & 8 & 11 & 3 & 2 & 51 & 336 & 15.18 \\
\hline \multirow{4}{*}{ Region } & Liaoning province & 5 & 8 & 8 & 10 & 7 & 1 & 0 & 39 & 200 & 19.50 \\
\hline & Jilin province & 6 & 4 & 8 & 9 & 9 & 3 & 2 & 41 & 200 & 20.50 \\
\hline & Heilongjiang province & 6 & 4 & 11 & 4 & 6 & 2 & 0 & 33 & 200 & 16.50 \\
\hline & Inner Mongolia Autonomous Region & 5 & 2 & 6 & 7 & 5 & 3 & 2 & 30 & 200 & 15.00 \\
\hline \multirow{3}{*}{ Breed } & Chinese White Rabbit & 9 & 6 & 14 & 8 & 10 & 4 & 1 & 52 & 249 & 20.88 \\
\hline & California Rabbit & 6 & 5 & 8 & 8 & 9 & 1 & 1 & 38 & 250 & 15.20 \\
\hline & New Zealand Rabbit & 7 & 7 & 11 & 14 & 8 & 4 & 2 & 53 & 301 & 17.61 \\
\hline Total & & 22 & 18 & 33 & 30 & 27 & 9 & 4 & 143 & 800 & 17.88 \\
\hline
\end{tabular}

1.56 -fold increase $(\mathrm{OR}=1.56,95 \% \mathrm{CI}=1.06-2.29, P<0.024)$ compared to Chlamydia infection in male (Table 2).

\section{Discussion}

Chlamydia spp. are gram-negative bacteria that cause various significant diseases in humans and animals all over the world, leading to health problems and serious economic losses. In the present study, the overall seroprevalence of Chlamydia infection in domestic rabbits was $17.88 \%$, which was higher than that observed previously in cats (5.9\%) [7], dogs (12.1\%) [15], pet birds (10.80\%) [16], pigeon (6.80\%) [17], red deer $(9.60 \%)$ [18], cattle $(4.75 \%)$ [19] and dairy cattle (7.25\%) [20], but lower than in sows (62.70\%) [21], wild boar (63.60\%) [22], and feral Canada geese (93.80\%) [23] using the same serological test (IHA) in China. Differences in Chlamydia seroprevalence are likely due to differences in animal welfares, animal categories, climates, and husbandry practices. Today, there is an increasing demand for rabbit meat in China, and the safety and sanitary quality of meat pose a potential threat to local residents and other people in exported regions. Therefore, it is important to understand the extent of Chlamydia infection in domestic rabbits in China.

In the present study, Chlamydia seroprevalence in juvenile rabbits (19.83\%) was higher than that in adult rabbits (15.18\%), but the differences were not statistically significant $(P>0.05)$, which was consistent with the previous study [14]. And the reason why juveniles were more susceptible than adults and subadults may be that the juveniles had lower immunity compared with adults. Moreover, the Chlamydia seroprevalence between male and female rabbits was significantly different $(P<0.05)$, implying that gender is a crucial factor for Chlamydia infection in domestic rabbits.

This study revealed that gender is a significant risk factor for Chlamydia prevalence in domestic rabbits, with females having a higher seroprevalence than males. The Chlamydia seroprevalence in male and female domestic rabbit was $14.11 \%$ and $20.34 \%$, respectively. This tendency is consistent with the studies of Tibetan pigs (females, $17.61 \%$; males, $12.72 \%$ ) [24] and also concurs with that observed in pet parrots (females, 41.22\%; males, 30.06\%) [14], in wild boars in Germany (females, 83.3\%; males, 42.9\%) [25] and Italy (females, 45.95\%; males, 38.8\%) [22]. Females and males were exposed to Chlamydia in the same environment, and in our study females are more sensitive to the pathogen than males for each of the breeds in each region group, which may be due to variation in immune response or antibody persistence rates between females and males.

In the present study, there was no significant difference in Chlamydia seroprevalence in rabbits in different provinces $(P>0.05)$. The statistically similar seropositivity suggests that the pathogen could be mostly transmitted by the direct contact route in domestic rabbits, which is little affected by environmental change. Thus, further studies are needed to illuminate the potential effect of Chlamydia on the geographical origin of domestic rabbits.

Chlamydia has been reported to be associated with abortion in horses, rabbits, guinea pigs, mice, and pigs [26]. More importantly, Chlamydia can cause devastating consequences of infertility, ectopic pregnancy, chronic pelvic 
pain, or even abortion in humans $[8,27]$, especially for people exposed to Chlamydia infection by working with rabbits, which increased the possibility to be infected [14, 28]. Thus, further studies are needed to illuminate the potential effect of Chlamydia on reproduction of domestic rabbits. Moreover, serological investigation of Chlamydia infection in human (particularly pregnant women) working in rabbit farms in northeast China is also warranted.

Among different breeds of domestic rabbits, Chlamydia seroprevalence in Chinese White Rabbit (20.88\%) was higher than that in New Zealand Rabbit (17.61\%) and California Rabbit (15.20\%), but the differences were not statistically significant, which was consistent with the previous study, and the animal species factor was calculated and was found to be of no significance [29]. Further investigations should be studied about the correlation of Chlamydia infection with the animal species.

In conclusion, the results of the present study revealed that Chlamydia infection in domestic rabbits is highly prevalent in northeast China, but this severe situation has been neglected in the past. Therefore, it is important to carry out integrated control strategies and measures to prevent and control Chlamydia infection in domestic rabbits in China. This is the first report of Chlamydia seroprevalence in domestic rabbits in China.

\section{Conflict of Interests}

The authors declare that they have no competing interests.

\section{Acknowledgments}

Project support was provided by study on the distribution of animal parasitic helminths and molecular taxonomy of Tianzhu White Yak (31460659) and Quality Inspection Special Public Welfare Industry Research (201410061).

\section{References}

[1] D. Longbottom and L. J. Coulter, "Animal chlamydioses and zoonotic implications," Journal of Comparative Pathology, vol. 128, no. 4, pp. 217-244, 2003.

[2] G. Rohde, E. Straube, A. Essig, P. Reinhold, and K. Sachse, “Chlamydial zoonoses," Deutsches Ärzteblatt International, vol. 107, no. 10, pp. 174-180, 2010.

[3] M. Eidson, "Psittacosis/avian chlamydiosis," Journal of the American Veterinary Medical Association, vol. 221, no. 12, pp. 1710-1712, 2002.

[4] G. Entrican and N. M. Wheelhouse, "Immunity in the female sheep reproductive tract," Veterinary Research, vol. 37, no. 3, pp. 295-309, 2006.

[5] Y. Pannekoek, C. Visser, B. Duim, and E. R. Heddema, "Chlamydophila psittaci infections in The Netherlands," Drugs of Today, vol. 45, pp. 151-157, 2009.

[6] K. Verminnen and D. Vanrompay, "Chlamydophila psittaci infections in Turkeys: overview of economic and zoonotic importance and vaccine development," Drugs of Today, vol. 45, pp. 147-150, 2009.
[7] S.-M. Wu, S.-Y. Huang, M.-J. Xu, D.-H. Zhou, H.-Q. Song, and X.-Q. Zhu, "Chlamydia felis exposure in companion dogs and cats in Lanzhou, China: a public health concern," BMC Veterinary Research, vol. 9, article 104, 2013.

[8] K. M. Osman, H. A. Ali, J. A. Eljakee, M. M. Gaafar, and H. M. Galal, "Antimicrobial susceptibility and molecular typing of multiple Chlamydiaceae species isolated from genital infection of women in Egypt," Microbial Drug Resistance, vol. 18, no. 4, pp. 440-445, 2012.

[9] K. M. Osman, H. A. Ali, J. A. ElJakee, and H. M. Galal, "Chlamydiaceae in riverine buffalo (Bubalus bubalis) and cows (Bos taurus) in Egypt with and without signs of reproductive disease," New Zealand Veterinary Journal, vol. 60, no. 4, pp. 228 233, 2012.

[10] D. S. A. Beeckman and D. C. G. Vanrompay, "Zoonotic Chlamydophila psittaci infections from a clinical perspective," Clinical Microbiology and Infection, vol. 15, no. 1, pp. 11-17, 2009.

[11] Y. F. Chen, X. P. Xie, and S. K. Sun, "The present situation and development countermeasures of Chinese rabbit industry," Modern Agriculture, vol. 12, pp. 6-7, 2010 (Chinese).

[12] E. V. Boiko, A. L. Pozniak, D. S. Maltsev, A. A. Suetov, and I. V. Nuralova, "Chronic ocular Chlamydia trachomatis infection in rabbits: clinical and histopathological findings in the posterior segment," Investigative Ophthalmology \& Visual Science, vol. 55, no. 2, pp. 1176-1183, 2014.

[13] Q. Chen, X. Gong, F. Zheng, X. Cao, Z. Li, and J. Zhou, "Seroprevalence of Chlamydophila abortus infection in yaks (Bos grunniens) in Qinghai, China," Tropical Animal Health and Production, vol. 46, no. 3, pp. 503-507, 2014.

[14] N. Z. Zhang, X. X. Zhang, D. H. Zhou et al., "Seroprevalence and genotype of Chlamydia in pet parrots in China," Epidemiology \& Infection, vol. 143, no. 1, pp. 55-61, 2015.

[15] Y. M. Tian, J. F. Cao, D. H. Zhou et al., "Seroprevalence and risk factors of Chlamydia infection in dogs in Southwestern China," Acta Tropica, vol. 130, no. 1, pp. 67-70, 2014.

[16] W. Cong, S. Y. Huang, X. X. Zhang et al., "Chlamydia psittaci exposure in pet birds," Journal of Medical Microbiology, vol. 63, no. 4, pp. 578-581, 2014.

[17] T. Stenzel, D. Pestka, and D. Choszcz, "The prevalence and genetic characterization of Chlamydia psittaci from domestic and feral pigeons in Poland and the correlation between infection rate and incidence of pigeon circovirus," Poultry Science, vol. 93, no. 12, pp. 3009-3016, 2014.

[18] A. di Francesco, M. Donati, S. Nicoloso et al., "Chlamydiosis: Seroepidemiologic survey in a red deer (Cervus elaphus) population in Italy," Journal of Wildlife Diseases, vol. 48, no. 2, pp. 488-491, 2012.

[19] K. Wilson, D. Sammin, S. Harmeyer, M. Nath, M. Livingstone, and D. Longbottom, "Seroprevalence of chlamydial infection in cattle in Ireland," The Veterinary Journal, vol. 193, no. 2, pp. 583585, 2012.

[20] D. H. Zhou, F. R. Zhao, H. Y. Xia et al., "Seroprevalence of chlamydial infection in dairy cattle in Guangzhou, Southern China," Irish Veterinary Journal, vol. 66, no. 1, article 2, 2013.

[21] X. X. Zhang, R. C. Li, G. H. Liu et al., "High seroprevalence of Chlamydia infection in sows in Hunan province, subtropical China," Tropical Animal Health and Production, vol. 46, no. 4, pp. 701-704, 2014.

[22] A. di Francesco, M. Donati, F. Morandi et al., "Seroepidemiologic survey for Chlamydia suis in wild boar (Sus scrofa) populations in Italy," Journal of Wildlife Diseases, vol. 47, no. 3, pp. 709-712, 2011. 
[23] V. Dickx, I. D. Kalmar, P. Tavernier, and D. Vanrompay, "Prevalence and genotype distribution of Chlamydia psittaci in feral Canada geese (Branta canadensis) in Belgium," Vector-Borne and Zoonotic Diseases, vol. 13, no. 6, pp. 382-384, 2013.

[24] N.-Z. Zhang, D.-H. Zhou, X.-C. Shi et al., "First report of chlamydiaceae seroprevalence in Tibetan pigs in Tibet, China," Vector-Borne and Zoonotic Diseases, vol. 13, no. 3, pp. 196-199, 2013.

[25] H. Hotzel, A. Berndt, F. Melzer, and K. Sachse, "Occurrence of Chlamydiaceae spp. in a wild boar (Sus scrofa L.) population in Thuringia (Germany)," Veterinary Microbiology, vol. 103, no. 1-2, pp. 121-126, 2004.

[26] K. Schautteet and D. Vanrompay, "Chlamydiaceae infections in pig," Veterinary Research, vol. 42, article 29, 2011.

[27] A. C. Ikeme, H. U. Ezegwui, L. C. Ikeako, I. Agbata, and E. Agbata, "Seroprevalence of Chlamydia trachomatis in Enugu, Nigeria," Nigerian Journal of Clinical Practice, vol. 14, no. 2, pp. 176-180, 2011.

[28] D. Baud, L. Regan, and G. Greub, "Emerging role of Chlamydia and Chlamydia-like organisms in adverse pregnancy outcomes," Current Opinion in Infectious Diseases, vol. 21, no. 1, pp. 70-76, 2008.

[29] K. M. Osman, H. A. Ali, J. A. Eljakee, and H. M. Galal, "Prevalence of Chlamydophila psittaci infections in the eyes of cattle, buffaloes, sheep and goats in contact with a human population," Transboundary and Emerging Diseases, vol. 60, no. 3, pp. 245-251, 2013. 


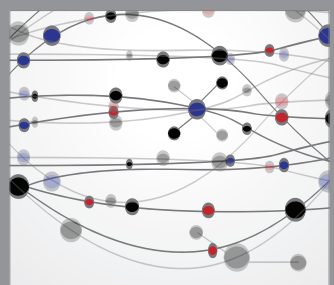

The Scientific World Journal
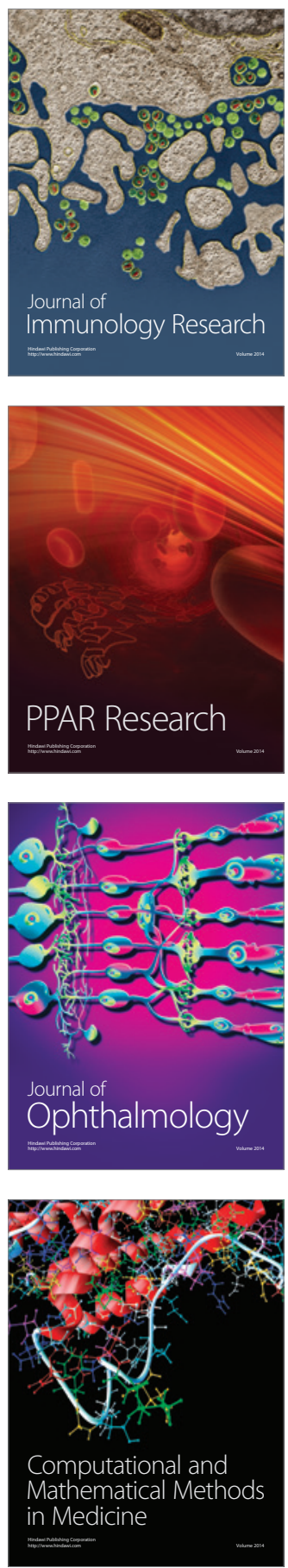

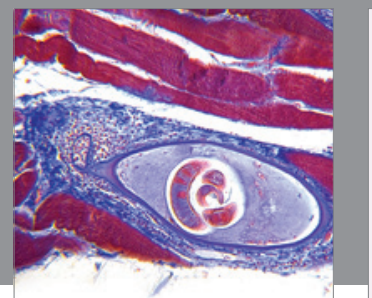

Gastroenterology

Research and Practice
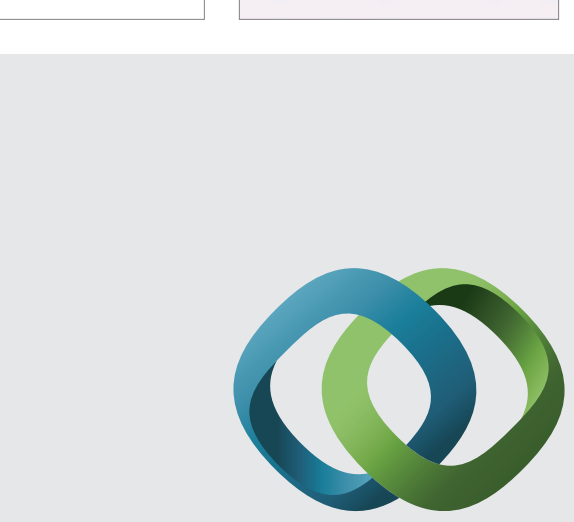

\section{Hindawi}

Submit your manuscripts at

http://www.hindawi.com
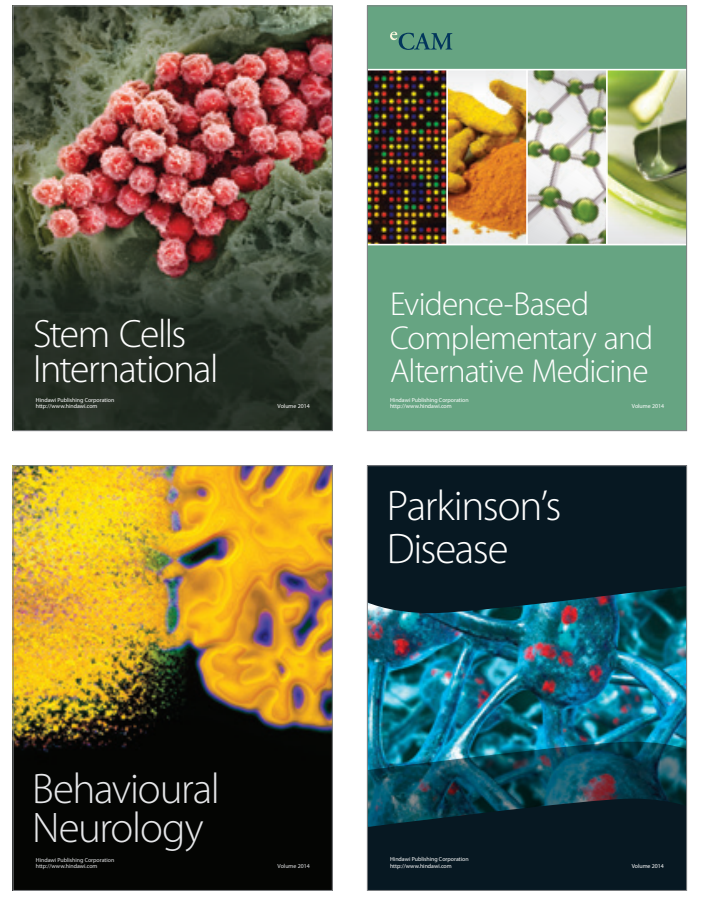
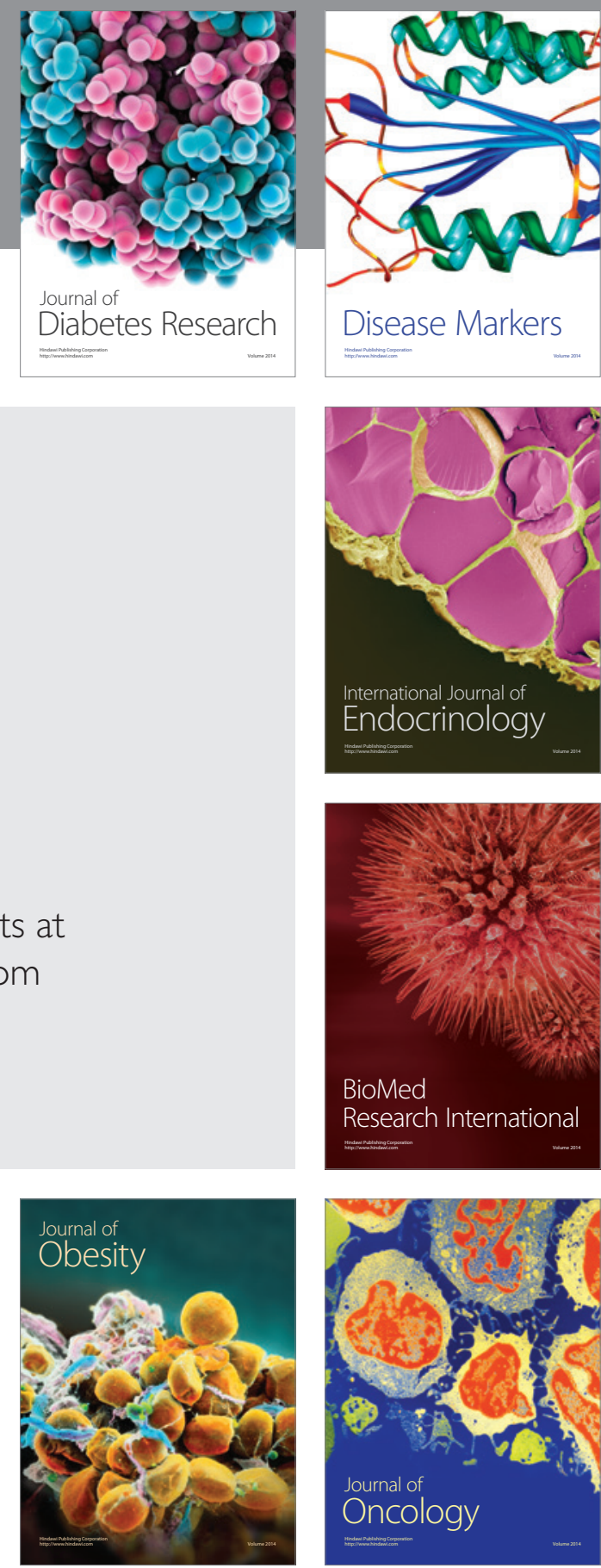

Disease Markers
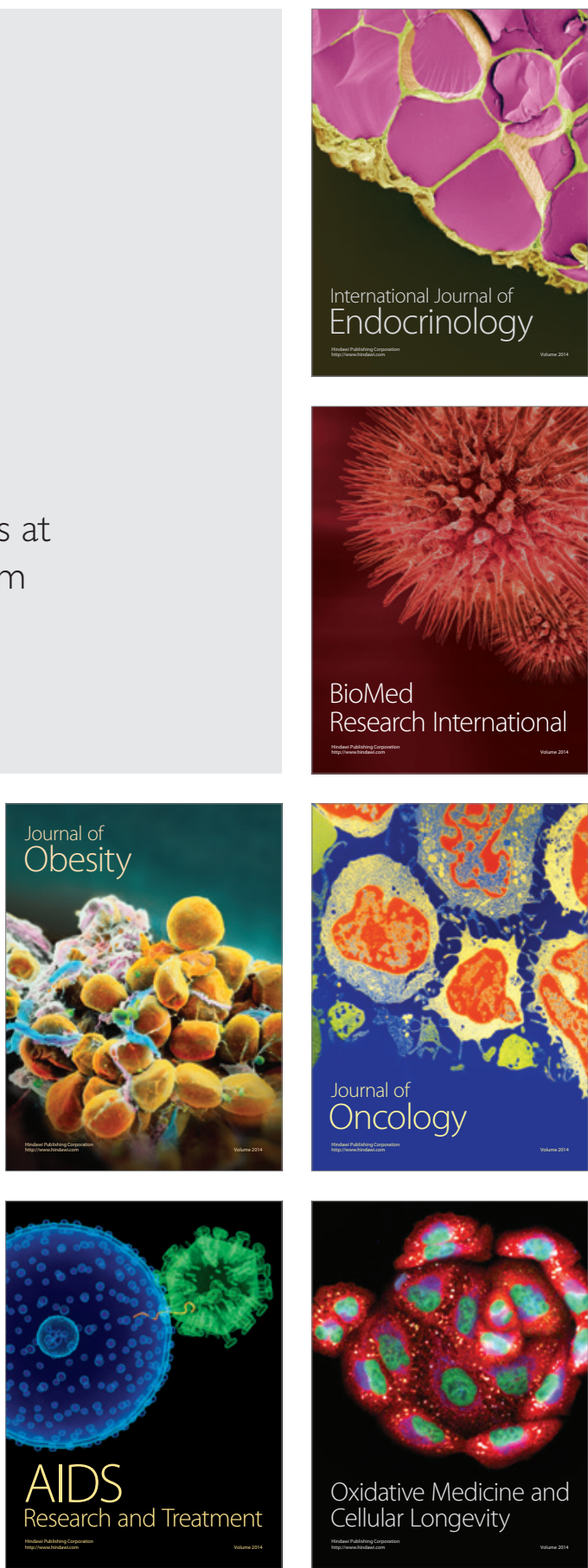